The IJA is a peer-reviewed open-access, electronic journal, freely available without charge to users

\title{
Effects of acute ammonia exposure and post-exposure recovery on nonspecific immunity in Clam Cyclina sinensis
}

\author{
Hongxing $\mathrm{Ge}^{1,2,3}$, Jialing Liu, ${ }^{1}$, Qian $\mathrm{Ni}^{1}$, Fei Wang ${ }^{1}$ \\ Zhiguo Dong ${ }^{1,2,3}$ \\ 1 Jiangsu Key Laboratory of Marine Bioresources and Environment, Jiangsu Ocean \\ University, Lianyungang, Jiangsu Province 222005, China \\ 2 Jiangsu Key Laboratory of Marine Biotechnology, Jiangsu Ocean University, \\ Lianyungang, Jiangsu Province 222005, China \\ ${ }^{3}$ Co-Innovation Center of Jiangsu Marine Bio-industry Technology, Jiangsu Ocean \\ University, Lianyungang, Jiangsu Province 222005, China
}

Keywords: Ammonia; Cyclina sinensis; Exposure; Immune enzyme activity; Recovery

\begin{abstract}
This study aimed to assess the toxicity of ammonia on clam Cyclina sinensis and the post-exposure recovery. With increased exposure to TAN, the alkaline phosphatase (AKP) activities after exposure showed a trend of growing initially and subsequently decreasing, whereas the AKP activities after post-exposure recovery showed an increasing trend. The AKP activities after post-exposure recovery were significantly higher than those in control. The acid phosphatase (ACP) activities in T1 and T2 after post-exposure recovery were higher than those in the control, whereas the ACP activities in T3, T4, and T5 after postexposure recovery were significantly higher than those in the control. The lysozyme (LZM) activities in T1 and T2 after exposure were significantly higher than those in control, whereas the LZM activities in T3, T4, and T5 after exposure were significantly lower than those in the control. Overall, ACP and LZM in the clams exposed to a low level of TAN ( $\leq 40 \mathrm{mg} / \mathrm{L}$ ) can recover to the normal levels completely. However, a $48 \mathrm{~h}$ recovery period scarcely seems adequate to compensate for AKP, ACP, and LZM activities in the clams exposed to a high level of TAN ( $>40 \mathrm{mg} / \mathrm{L}$ ).
\end{abstract}

\footnotetext{
* Corresponding author. e-mail: dzg2004@163.com; First author, e-mail: hongxinggeliu@163.com
} 


\section{Introduction}

It is well-known that ammonia is one of the most severe environmental pollutants in aquaculture systems (Ge et al., 2021a). Ammonia usually exists in two chemical forms: ionized ammonium $\left(\mathrm{NH}_{4}{ }^{+}\right)$and unionized ammonia $\left(\mathrm{NH}_{3}\right)$. As $\mathrm{NH} 3$ is of strong fat solubility, it can damage gills and other tissues of marine animals to varying extents (Foss et al., 2009). Studies have demonstrated that ammonia accumulation usually has a negative effect on marine animals (Florence et al., 2015; Gao et al., 2016). In severe cases, the mortality of cultured marine animals can increase sharply (Wang et al., 2012). Therefore, ammonia has been identified as one of the most critical limiting factors for marine animals (Ge et al., 2021b).

There is growing evidence that environmental pollutants can cause immune system disorder in marine animals (Ge et al., 2021b; Sreekakula et al., 2019). Diverse studies have indicated that alkaline phosphatase (AKP), acid phosphatase (ACP), and lysozyme (LZM) play key functions in the nonspecific immune system (Chen et al., 2019; Liu et al., 2004; Yuan et al., 2020). As important phosphatase enzymes, ACP and AKP can resist pathogens by releasing attached phosphoryl groups from pathogenic bacteria and storing them in lysosomes (Long et al., 2021; Xu et al., 2020). LZM is one of the most important regulators of innate immune responses, which can attack the peptidoglycan layer of the bacterial cells (Bayarri et al., 2014). AKP, ACP, and LZM, which are involved in various metabolic processes, have been determined to be indicators to reveal the stress responses of marine animals to the growth environment (Chen et al., 2019; Xu et al., 2020). Effects of ammonia on immune responses of marine animals have drawn a lot of attention recently because immunity variations under environmental stresses are bound up with the occurrence and development of fish diseases (Ge et al., 2021a; Zhang et al., 2019). However, studies on the acute toxicity of ammonia on nonspecific immunity in mollusk are still essential to reveal the sensitivity to this relevant contaminant.

The clam Cyclina sinensis is an economically important marine clam ( $\mathrm{Ni}$ et al., 2020). And the clam distributes widely in coastal areas of East Asia. Because of its rapid growth rate, delicious taste, and resistance to diseases, clam farming contributes a lot to sustainable clam industry development in China (Ni et al., 2021). However, environmental stresses can inhibit mollusks' physiological and immune systems, which can severely influence marine animals (Ching et al., 2009). Previous studies have revealed that toxic ammonia affects the physiological reactions in marine animals (Gao et al., 2016; Yang et al., 2010). Nevertheless, the actual toxic threshold varies greatly in different marine animals (Foss et al., 2009). Furthermore, whether the damage caused by ammonia exposure can recover to the original level remains unclear (Zhang et al., 2019).

Generally, the first toxicological evaluation of a specific combination "organism + toxicant" is to determine the average lethal concentration levels (LC50) (Zhang et al., 2019). It is well accepted that LC50 value can be calculated by acute toxicity experiments of short duration, typically 48 to $96 \mathrm{~h}$ (Acar et al., 2018). However, traditional toxicological researches mainly concentrate on the dose-effect relationship between the pollutants and organisms at the given exposure time (Lia et al., 2014). How the poisonous effect of pollutants varies after exposure is often ignored (Chen and Guo, 2015). Therefore, the toxic effect during the post-exposure period should also be taken into consideration to evaluate the toxic effect of pollutants on organisms comprehensively. Within this context, firstly, we determined the median lethal concentrations (LC50) and safe concentration (SC) of TAN and $\mathrm{NH} 3$ for $C$. sinensis. And then, we assess the effect of acute ammonia exposure and post-exposure recovery on nonspecific immunity in $C$. sinensis. The results may help to reveal the underlying relationship between immune response and ammonia toxicity.

\section{Materials and Methods}

Clam source, nursery and feeds

Healthy clam $C$. sinensis (average body weight $3.38 \pm 0.21 \mathrm{~g}$ ) were obtained from Lianyungang Zhongchuang Aquaculture Company. Test clam C. sinensis were acclimated 
in polyvinyl chloride tanks $(45 \mathrm{~cm} \times 30 \mathrm{~cm} \times 40 \mathrm{~cm})$ containing $30 \mathrm{~L}$ well-aerated sand-filtered seawater (temperature: $24^{\circ} \mathrm{C}$, salinity: $21 \mathrm{ppt}, \mathrm{pH}: 8.0$, dissolved oxygen: $5.3 \mathrm{mg} / \mathrm{L}$, and TAN $<0.01 \mathrm{mg} / \mathrm{L}$ ) for ten days before the experiment. Only healthy $C$. sinensis of uniform size without pathological signs were selected as test subjects. During the adaptation period, test clams were fed twice with microalgae. Feeding was ceased one day before the experiment.

The stock solution of high purity ammonium chloride $\left(\mathrm{NH}_{4} \mathrm{Cl}\right)(10 \mathrm{~g} / \mathrm{L})$ was diluted to the desired concentration of total ammonia (TAN). During the exposure test, the TAN level was measured every $12 \mathrm{~h}$ with a spectrophotometer (DR 3800, Hach) followed by Ge et al. (2021). To maintain the level of TAN, $100 \%$ of seawater was renewed every $12 \mathrm{~h}$, and seawater contained the designed concentration of TAN while guaranteeing the other water quality stabilization. During the exposure test, the $\mathrm{pH}$ was monitored every $12 \mathrm{~h}$ using a portable $\mathrm{pH}$ meter (PHB-5, Leica, China), and the level was maintained within the range of the control group $(8.0 \pm 0.3)$ with diluted $\mathrm{HCl}$ and, or $\mathrm{KOH}$ (Egnew et al., 2019).

\section{Experimental design}

Preliminary experiment

Preliminary experiments were conducted to determine the LC50 concentration of ammonia for $C$. sinensis. Firstly, using the probit analysis method (Acar et al., 2018; Fossog et al., 2013; Ge et al., 2021b), we evaluated the minimum safe dose (0 lethal concentration, MSD) and maximum lethal dose (100\% lethal concentration, MLD) were 75.02 and $375.28 \mathrm{mg} / \mathrm{L}$, respectively. Then, the desired levels of TAN were set as 75.02, $125.06,175.1,225.14,275.18,325.22$, and $375.28 \mathrm{mg} / \mathrm{L}$. Each treatment was conducted in triplicate with a density of 20 individuals per tank. The clam, which cannot close the double shell and cannot respond to stimuli, was defined as a dead clam. The dead clam was removed from the tanks every six $h$, and record the numbers of dead clams. Finally, the $\mathrm{LC}_{50}$ for clam $C$. sinensis and the confidence limit of $95 \%$ was calculated with Karber's method (Ge et al., 2021a). The level of $\mathrm{NH}_{3}$ was calculated with TAN, $\mathrm{pH}$, and temperature of the equation: $\mathrm{NH}_{3}=\mathrm{TAN} /(10(\mathrm{p} \mathrm{Ka}-\mathrm{pH})+1)(\mathrm{Ge}$ et al., 2021a).

\section{Ammonia- $N$ exposure test}

According to the $48-\mathrm{h} \mathrm{LC}_{50}$ of TAN for clam C. sinensis we determined above, 360 clams were selected and divided into six groups with 18 tanks (six groups of three replicate tanks, 20 individuals per tank) randomly, and exposed to 0 (control), 20 (T1), 40 (T2), 60 (T3), 80 (T4) and $100 \mathrm{mg}$ (T5) TAN /L, respectively. As marine animals exposed to ammonia for $48 \mathrm{~h}$ can lead to severe toxic effects (Ge et al. 2021), the clam was exposed to ammonia for $48 \mathrm{~h}$. The preliminary experimental results show that some immune parameters in low ammonia nitrogen treatment could return to their normal levels in $48 \mathrm{~h}$. Therefore, at the cessation of ammonia exposure, the clams were moved to the control conditions for $48 \mathrm{~h}$ (the recovery group). During the test, no feed was supplied.

\section{Hepatopancreas collection and enzyme activity assay}

Five individuals per tank were randomly collected after exposure and post-exposure recovery in each group. As the main target organs impaired by ammonia is the hepatopancreas (Ge et al., 2021b), the clam C. sinensis were decontaminated with $70 \%$ ethanol and then dissected to obtain hepatopancreas tissue immediately with sterile scissors. The enzyme activities were determined using diagnosis kits for AKP, ACP, and LZM according to the protocol recommended by the manufacturer (Nanjing Jiancheng Bioengineering Institute, Nanjing) according to the protocol recommended by the manufacturer (Ge et al., 2021a).

\section{Statistical analysis}

Values were calculated using SPSS 18.0 software. Results were expressed as means \pm standard deviation. One-way analysis of variance (ANOVA) followed by Duncan's test was conducted to assess the significant differences among treatments (Ge et al., 2019). P < 0.05 was considered to be statistically different. 


\section{Results}

\section{$L C_{50}$ and SC}

Exposed to ammonia, the mortality of $C$. sinensis increased along with the increasing concentration of ammonia (Table 1). The $\mathrm{LC}_{50}$ in 24, 48, 72, and 96h of TAN were 181.30, $118.17,105.03$, and $80.72 \mathrm{mg} / \mathrm{L}$, respectively. The $\mathrm{LC}_{50}$ in $24,48,72$, and $96 \mathrm{~h}$ of $\mathrm{NH} 3$ were $9.00,5.87,5.22$, and $4.01 \mathrm{mg} / \mathrm{L}$, respectively. The $96 \mathrm{~h}$ safe concentration of TAN and $\mathrm{NH}_{3}$ for the clams was 8.07 and 0.40 , respectively.

Table 1 The LC50 and safe concentration (SC) for clam C. sinensis

\begin{tabular}{lllll}
\hline Time & LC 50 of TAN $(\mathrm{mg} / \mathrm{L})$ & $\mathrm{LC}_{50}$ of $\mathrm{NH}_{3}(\mathrm{mg} / \mathrm{L})$ & $\mathrm{SC}$ of TAN $(\mathrm{mg} / \mathrm{L})$ & $\mathrm{SC}$ of $\mathrm{NH}_{3}(\mathrm{mg} / \mathrm{L})$ \\
\hline 24 & 181.30 & 9.00 & 18.13 & 0.90 \\
48 & 118.17 & 5.87 & 11.82 & 0.59 \\
72 & 105.03 & 5.22 & 10.50 & 0.52 \\
96 & 80.72 & 4.01 & 8.07 & 0.40 \\
\hline
\end{tabular}

Effect of acute ammonia exposure on nonspecific immunity enzyme activities in C. sinensis

The AKP activities in the hepatopancreas tissue of clam $C$. sinensis after exposure showed a trend of increasing firstly and then decreasing along with the increasing concentration of ammonia (Figure 1). The AKP activities in the groups of exposure were significantly higher than those in the control $(P<0.05)$. The AKP activities in the recovery groups showed an increasing trend along with the increasing concentration of ammonia, and the AKP activities in the recovery groups were significantly higher than those in the control $(P<0.05)$. The AKP activities in the exposure groups were significantly higher than those in the group of post-exposure recovery $(P<0.05)$.

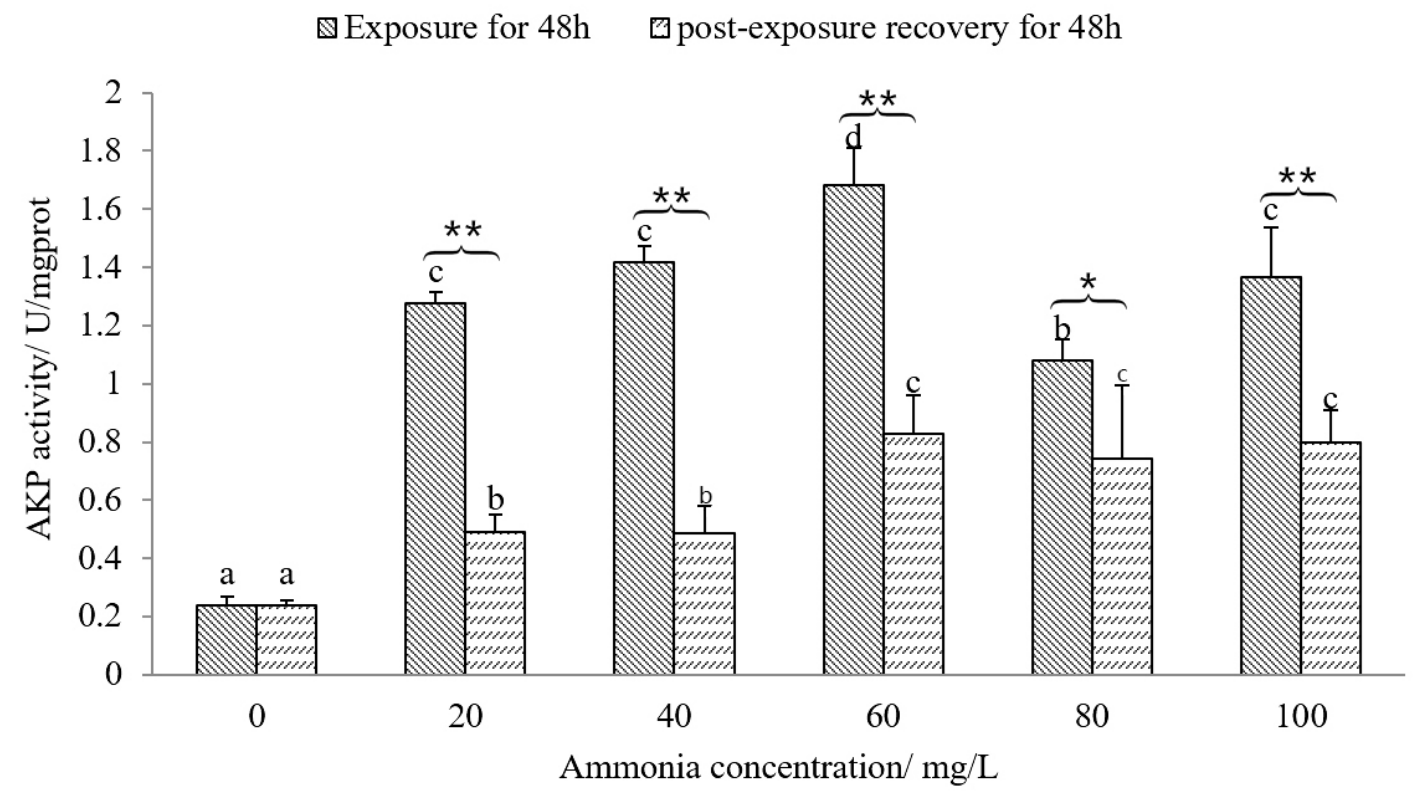

Figure 1 The AKP activity in the hepatopancreas tissue of clam $C$. sinensis after exposed to ammonia. The same lowercase means no significant difference in different exposure concentration of ammonia at the same time (exposure for $48 \mathrm{~h}$ or post-exposure recovery for $48 \mathrm{~h}$ ), otherwise significant differences $(P<0.05)$. Significant differences in the same concentration of ammonia between exposure for $48 \mathrm{~h}$ and post-exposure recovery for $48 \mathrm{~h}$ are indicated by asterisks $(* P<0.05, * *$ $P<0.01)$. 


\section{Effect of salinity on IBR of the clams reared in different salinities}

The IBR values were calculated using the gill enzyme activities (LZM, NKA, SOD and GPT) (Table 2). The IBR values of the clams had a tendency to increase with salinity decreased and they were $11.28,3.40$ and 2.85 in $10 \%$, $20 \%$ ond $30 \%$, respectively.

The biomarker star chart for IBR of the clams reared in different salinities (LZM, NKA, SOD and GPT in gills) as is shown in Figure 3 and the IBR value of the clams under the salinity stress was the area formed by each radius coordinate. In group $10 \%$, GPT made the biggest contributor to IBR, followed by LZM, NKA and SOD. In group 20\%o, LZM made the minimum contributor, followed by SOD and the contributions of GPT and NKA were similar. In group $20 \%$, GPT made the minimum contributor, and the other three indicators were similar.

As shown in Figure 2, the ACP activities after exposure increased significantly along with the increasing concentration of ammonia. The AKP activities in the groups of exposure were significantly higher than those in the control $(P<0.05)$. The ACP activities in the recovery groups increased along with the exposure concentration of ammonia, whereas they were significantly lower than in the groups of exposure $(P<0.05)$. The ACP activities in T1 and T2 after recovery were higher than those in the control $(P>0.05)$, whereas the ACP activities in T3, T4, and T5 after recovery were significantly higher than those in the control $(P<0.05)$.

The LZM activities after exposure showed a trend of increasing firstly and then decreasing along with the increasing concentration of ammonia (Figure 3). The LZM activities in T1 and T2 after exposure were significantly higher than those in the control $(P<0.05)$, whereas the LZM activities in T3, T4, and T5 after exposure were significantly lower than those in the control $(P<0.05)$. The LZM activity in T1 after recovery was significantly higher $(P<0.05)$ than that in the control, whereas the LZM activity in T3, T4, and T5 after recovery was significantly lower $(P<0.05)$.

\section{$\Delta$ Exposure for $48 \mathrm{~h} \quad \square$ post-exposure recovery for $48 \mathrm{~h}$}

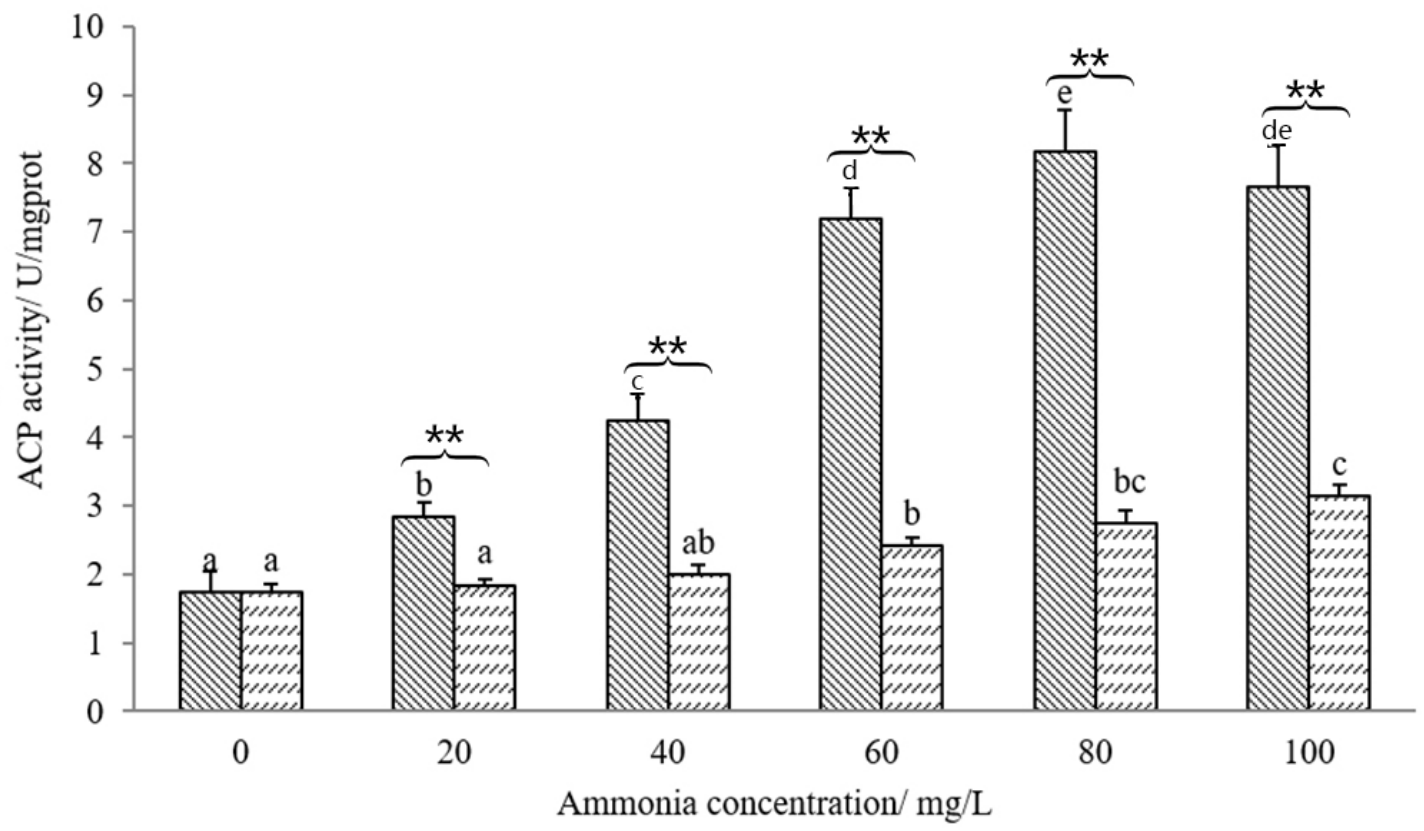

Figure 2 The ACP activity in the hepatopancreas tissue of clam $C$. sinensis after exposed to ammonia.The same lowercase means no significant difference in different exposure concentration of ammonia at the same time (exposure for $48 \mathrm{~h}$ or post-exposure recovery for $48 \mathrm{~h}$ ), otherwise significant differences $(P<0.05)$. Significant differences in the same concentration of ammonia between exposure for $48 \mathrm{~h}$ and post-exposure recovery for $48 \mathrm{~h}$ are indicated by asterisks $(* P<0.05$, $* * P<0.01)$. 


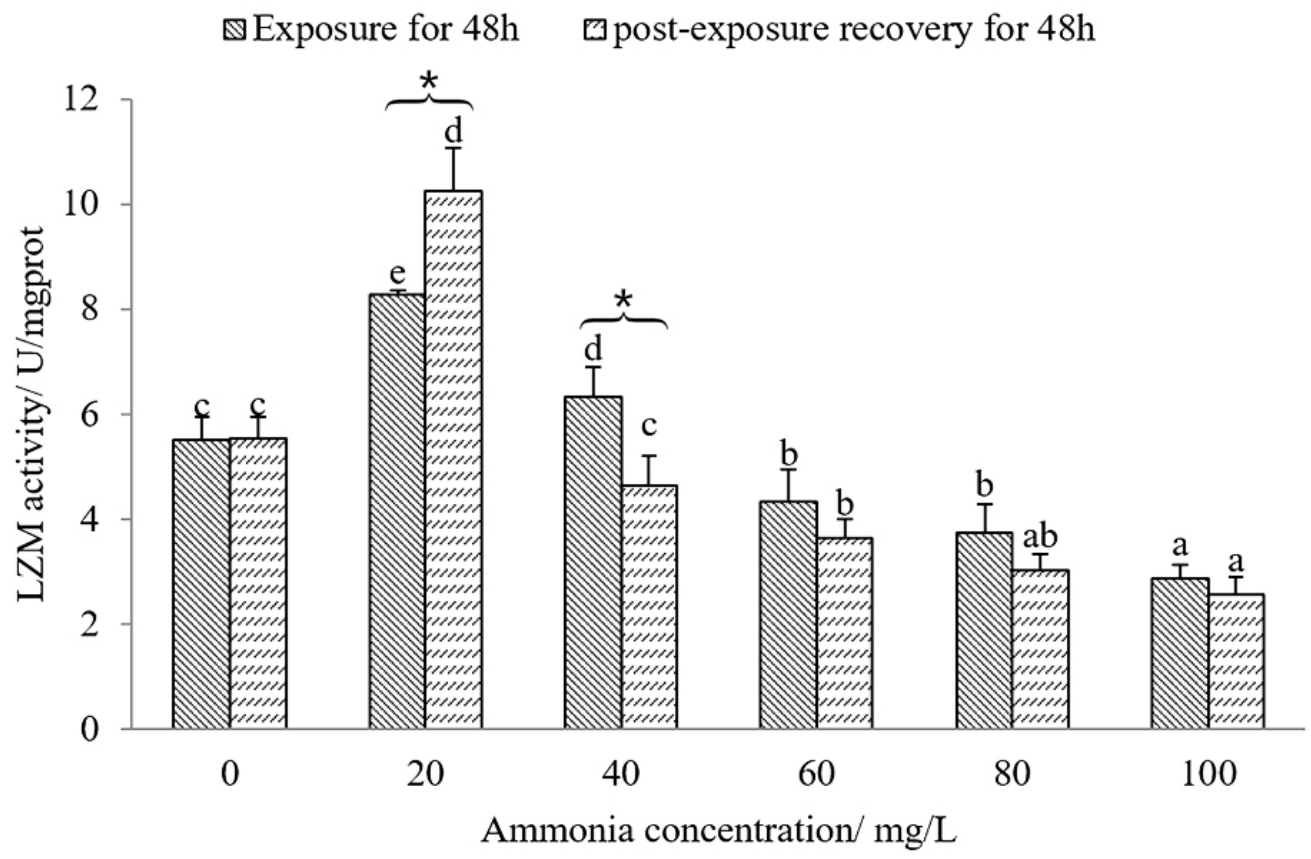

Figure 3 The LZM activity in the hepatopancreas tissue of clam C. sinensis after exposed to ammonia. The same lowercase means no significant difference in different exposure concentration of ammonia at the same time (exposure for $48 \mathrm{~h}$ or post-exposure recovery for $48 \mathrm{~h}$ ), otherwise significant differences $(P<0.05)$. Significant differences in the same concentration of ammonia between exposure for $48 \mathrm{~h}$ and post-exposure recovery for $48 \mathrm{~h}$ are indicated by asterisks $(* P<0.05, * *$ $P<0.01)$.

\section{Discussion}

Ammonia is harmful to marine animals (Florence et al., 2015; Lu et al., 2016). The toxic effect of ammonia on marine animals has attracted wide attention. Lots of studies have revealed the toxicity of ammonia to mollusks, such as Asian clam Corbicula fluminea (Zhang et al., 2019) and clam Ruditapes philippinarum (Cong et al., 2019). Ammonia accumulation in water may be a severe threat to marine animals (Ge et al., 2021b). In our present research, the $96 \mathrm{~h}$ safe concentration of TAN and $\mathrm{NH}_{3}$ for the clam was 8.07 and 0.40 , respectively. They are relatively higher than the SC of other marine animals, such as Scylla serrate (Romano and Zeng, 2007) and Litopenaeus vannamei (Lu et al., 2016). This is probably because the bivalve mollusks have shells and uniquely efficient mechanisms of detoxification metabolism (Zhang et al., 2019). Generally, the damage aggravated along with the increasing environmental stressor concentration (Ge et al., 2021b). Cong et al. (2017) reported that the clam $R$. philippinarum exposed to the level of $0.5 \mathrm{mg} / \mathrm{L}$ ammonia might suffer severe effects, including gill damage and neurotoxicity. James \& Diane reported that the LC50-96h for the survival of clam Spisula solidissima was $10.6 \mathrm{mg} / \mathrm{L}$ TAN and $0.53 \mathrm{mg} / \mathrm{L} \mathrm{NH}_{3}$ (James \& Diane, 2011). In the present study, the average lethal concentration levels increased along with the death time reduced. Elevated ammonia in water can accelerate the accumulation of ammonia uptake across the gill epithelium. However, the accumulation of ammonia in organisms usually causes very high ammonia levels in the body fluids, and even leads to death (Sreekakula et al., 2019).

AKP is a lysosomal enzyme and plays an essential role in the nonspecific immune system by catalyzing the hydrolysis of various phosphate-containing compounds in the alkaline environment (Gobi et al., 2016). In the present research, the AKP activities after exposed to ammonia showed a trend of increasing firstly and then decreasing. The result indicates that ammonia exposure can influence AKP activities in $C$. sinensis. Some previous studies have revealed that low levels of potentially environmental toxic pollutants, bacteria, or viruses could cause stimulatory effects on the immune system (Stebbing, 1982; Wai-San 
et al., 2011). This phenomenon is so-called "hormesis" (Stebbing, 1982). That's possible because environmental chemicals could inhibit or induce mRNA expression of innate immune-related genes and cytokines and further result in the change of immune enzymes, such as LZM and AKP (Jia et al., 2014; Rogers et al., 2013). In the present research, The AKP activities in the recovery groups showed an increasing trend along with the increasing concentration of ammonia, and the AKP activities in the recovery groups were significantly higher than those in the control. This indicated that AKP activity couldn't recover completely after being transferred to pristine seawater for $48 \mathrm{~h}$ for those exposed to a high level of ammonia. Compared to the group of exposure, the AKP activity in the group of postexposure recovery has recovered somewhat. However, it is more difficult for the clam exposed to a high level of ammonia (c $>40 \mathrm{mg} / \mathrm{L}$ ) to resume its original activity than those in a low level of ammonia ( $c \leq 40 \mathrm{mg} / \mathrm{L}$ ). This is probably because that the high level of potentially toxic agents caused immune system damage irreparably, or maybe it is because of a $48 \mathrm{~h}$ recovery insufficient recovery time (Yang et al., 2010).

ACP is one of the marker enzymes of macrophage lysosome in organisms, and ACP plays an essential role by destroying and eliminating foreign bodies in the clam nonspecific immunity (Xia and Wu, 2018). In the present study, the ACP activities in clam C. sinensis after exposure increased along with the increasing concentration of ammonia. The result showed that ammonia exposure could cause stimulatory effects on ACP activity. During the same recovery time, the ACP activities in the low level of ammonia (c $\leq 40 \mathrm{mg} / \mathrm{L}$ ) could resume their original level. Whereas the ACP activities in a high level of ammonia ( $c>$ $40 \mathrm{mg} / \mathrm{L}$ ) after post-exposure recovery were significantly higher than those in the control. This indicated that ACP activity in the clam which exposed to the lower concentration of ammonia (c $\leq 40 \mathrm{mg} / \mathrm{L}$ ) could recover in $48 \mathrm{~h}$. It is possibly because of overcompensation. The occurrence of overcompensation response of exceeding compensation after the organism suffered damaging stress (Xie et al., 2012).

As an important hydrolytic enzyme, LZM could kill bacteria by destroying their cell walls (Bayarri, 2014). Divers studies indicated that ammonia exposure could decrease humoral immune responses of aquatic animals, such as bacteriolytic (Yue et al., 2010). In the present research, the LZM activities after exposure showed a trend of increasing initially and subsequently decreasing along with the increasing concentration of ammonia. It indicated that a low level of ammonia exposure could cause stimulatory effects on LZM activity, whereas a high level of ammonia exposure could cause inhibitory effects on LZM activity. LZM is an essential regulator of innate immune responses, and high-level activity will help to destroy bacterial cells (Jash \& Kumar, 2014). Compared to the control, the LZM activity in T1 after post-exposure recovery, indicating that a low level of ammonia stress induces overcompensation (Xie et al., 2012). The LZM activity in T2 was lower, indicating that LZM activity can resume its original level. The LZM activities in groups exposed to the high level of ammonia (c $>40 \mathrm{mg} / \mathrm{L}$ ) after post-exposure recovery were significantly lower than those in the control, indicating that LZM activity in the clam can't recover from ammonia post-exposure recovery in $48 \mathrm{~h}$. This is possibly because that the mechanism of LZM synthesis in the clam exposed to a high level (c $>40 \mathrm{mg} / \mathrm{L}$ ) was inhibition, which might even lead to irreversible damage (Xu et al., 2020; Oliveira et al., 2018).

\section{Conclusion}

In conclusion, the LC50 of TAN for the clam C. sinensis after $24,48,72$, and $96 \mathrm{~h}$ were $181.30,118.17,105.03$, and $80.72 \mathrm{mg} / \mathrm{L}$, respectively. Chronic ammonia exposure for $48 \mathrm{~h}$ can cause a rise in AKP and ACP activities. However, it causes a reduction in LZM activity. After post-exposure recovery for $48 \mathrm{~h}$, activities of ACP and LZM in the clams exposed to a low level of ammonia ( $\leq 40 \mathrm{mg} / \mathrm{L}$ ) can recover to the normal levels completely, whereas a $48 \mathrm{~h}$ recovery period scarcely seems adequate to compensate for $A K P, A C P$, and LZM activities in the clams exposed to a high level of ammonia of TAN ( $>40 \mathrm{mg} / \mathrm{L})$. 


\section{Acknowledgements}

This research was supported by National key research and development projects (2019YFD0900403), China Agriculture Research System of MOF and MARA, the Priority and Academic Program Development of Jiangsu Higher Education Institutions (20KJB240002), Postdoctoral Fellowship of Lianyungang, and the Opening Foundation of Jiangsu Institute of Marine Resources Development (JSIMR202017). The funding bodies play a key role in the design of the study, the collection, analysis, and interpretation of data and in writing the manuscript.

\section{References}

Acar, Ü., Ínanan, B., Zemheri, F., Kesbic, O., and Yilmaz. S., 2018. Acute exposure to boron in Nile tilapia (Oreochromis niloticus): Median-lethal concentration (LC50), blood parameters, DNA fragmentation of blood and sperm cells. Chemosphere 213, 345-350.

https://doi.org/10.1016/j.chemosphere.2018.09.063

Bayarri, M., Oulahal, N., Degraeve P. and A. Gharsallaoui, 2014. Properties of lysozyme/low methoxyl (LM) pectin complexes for antimicrobial edible food packaging. J. Food Eng. 131, 18-25. https://doi.org/10.1016/j.jfoodeng.2014.01.013

Chen, J. and R.Guo, 2015. The process-dependent impacts of dimethoate on the feeding behavior of rotifer. Chemosphere 119, 318-325. https://doi.org/10.1016/j.chemosphere.2014.06.07

Chen, S., Yu, Y., Gao, Y., Yin, P., Tian, L., Niu, J. and Y. Liu, 2019. Exposure to acute ammonia stress influences survival, immune response andantioxidant status of pacific white shrimp (Litopenaeus vannamei) pretreated with diverse levels of inositol. Fish shellfish immune. 89, 248-256. https://doi.org/10.1016/j.fsi.2019.03.072

Ching, B.Y., Chew, S.F., Wong, W.P. and Y.K. Ip, 2009. Environmental ammonia exposure induces oxidative stress in gills and brain of Boleophthalmus boddarti (mudskipper). Aquat. Toxicol. 203-212. https://doi.org/10.1016/j.aquatox.2009.09.004

Cong, M., Wu, H., Cao, T., Ji, C. and J. Lv, 2019. Effects of ammonia nitrogen on gill mitochondria in clam Ruditapes philippinarum. Environ. Toxicol. Phar. 65, 46-52.

https://doi.org/10.1016/j.etap.2018.12.003

Cong, M., Wu, H., Yang, H., Zhao, J. and Lv J., 2017. Gill damage and neurotoxicity of ammonia nitrogen on the clam Ruditapes philippinarum. Ecotoxicology 26, 459-469. https://doi.org/10.1007/s10646-017-1777-4

Egnew, N., Renukdas, N., Ramena, Y., Yadav, A., Kelly A., Lochmann, R. and A. Sinha, 2019. Physiological insights into largemouth bass (Micropterus salmoides) survival during long-term exposure to high environmental ammonia, Aquat. Toxicol. 2017, 72-82. https://doi.org/10.1016/j.aquatox.2018.11.027

Florence, B., Béatrice, D., Sébastien, L., Laurent, B. and H. Joël, 2015. Storage of Pacific oysters Crassostrea gigas in recirculating tank: Ammonia excretion and potential nitrification rate. Aquacult. Eng. 64, 8-14. https://doi.org/10.1016/j.aquaeng.2014.11.007 Foss, A., Imsland, A.K., Roth, B., Schram, E. and S.O. Stefansson, 2009. Effects of chronic and periodic exposure to ammonia on growth and blood physiology in juvenile turbot (Scophthalmus maximus). Aquaculture 296, 45-50.

https://doi.org/10.1016/j.aquaculture.2009.07.013

Fossog, B., Antonio-Nkondjio, C., Kengne and P., F. Njiokou, 2013. Physiological correlates of ecological divergence along an urbanization gradient: Differential tolerance to ammonia among molecular forms of the malaria mosquito Anopheles gambiae. BMC Ecology 13(1):1. https://doi.org/10.1186/1472-6785-13-1

Gao, S., Ren, Y., Zhang, H., Pan, B. and H. Gao, 2016. Identification and expression analysis of IKB and NF-KB genes from Cyclina sinensis. Fish shellfish immune. 56, 427435. https://doi.org/10.1016/j.fsi.2016.07.035

Ge, H., Liang, X., Liu, J., Cui, Z., Guo, L., Li, L., Sun, Y. and Z. Dong, 2021a. Effects of acute ammonia exposure on antioxidant and detoxification metabolism in clam Cyclina 
sinensis. Ecotox. Environ. Safe. https://doi.org/10.1016/j.ecoenv.2021.111895

Ge, H., Ni, Q., Li, J., Li, J., Chen, Z. and F. Zhao, 2019. Integration of white shrimp (Litopenaeus vannamei) and green seaweed (Ulva prolifera) in minimum-water exchange aquaculture system. J. Appl. Phycol. 31(1): 1425-1432. https://doi.org/10.1007/s10811018-1601-4

Ge, H., Shi, J., Liu, J., Guo, L. and Z. Dong, 2021b. Combined analysis of mRNA-miRNA reveals the regulatory roles of miRNAs in the metabolism of clam Cyclina sinensis hepatopancreas during acute ammonia nitrogen stress. Aquacult. Res., 15683. https://doi.org/10.1111/are.15683

Gobi, N., Ramya, C., Vaseeharan, B., Malaikozhundan, B., Vijayakumar, S., Murugan, K. and G. Benelli, 2016. Oreochromis mossambicus diet supplementation with Psidium guajava leaf extracts enhance growth, immune, antioxidant response and resistance to Aeromonas hydrophila. Fish Shellfish Immun. 58, 572-583. https://doi.org/10.1016/j.fsi.2016.09.062

James A.F. and F.C. Diane, 2011. Toxicity of ammonia to surf clam (Spisula solidissima) larvae in saltwater and sediment elutriates. Mar Environ Res. 71: 189-194. https://doi.org/10.1016/j.marenvres.2011.01.002

Jash C. and G.S. Kumar, 2014. Binding of alkaloids berberine, palmatine and coralyne to lysozyme: a combined structural and thermodynamic study. RSC Adv. (4), 12514-12525. https://doi.org/10.1039/c3ra46053c

Jia, R., Cao, L.P., Du, J.L., Wang, J.H., Liu, Y. and G. Jeney, 2014. Effects of carbon tetrachloride on oxidative stress, inflammatory response and hepatocyte apoptosis in common carp (Cyprinus carpio), Aquat. Toxicol. 152, 11-19.

https://doi.org/10.1016/j.aquatox.2014.02.014

Lia, M., Yu, N., Qin, J.G., Li, E., Du, Z.Y. and L.Q. Chen, 2014. Effects of ammonia stress, dietary linseed oil and Edwardsiella ictaluri challenge on juvenile darkbarbel catfish Pelteobagrus vachelli. Fish Shellfish Immunol. 38, 158-165.

https://doi.org/10.1016/j.fsi.2014.03.015

Liu, S.L., Jiang, X.L., Hu, X.K., Gong, J., Hwang, H. and K. Mai, 2004. Effects of temperature on non-specific immune parameters in two scallop species: Argopecten irradians (Lamarck 1819) and Chlamys farreri (Jones \& Preston 1904). Aquacult. Res. 35 (7), 678-682. https://doi.org/10.1111/j.1365-2109.2004.01065.X

Liu, Y., Wang, Y., Zhang, J., Sun, L., Zhang, A., Torres, O.L., Guo, R. and J. Chen, 2017. An integrated assessment of ceftazidime and photoproducts on the feeding behavior of rotifers: From exposure to post-exposure. Ecotox. Environ. Safe. 144, 245-251. https://doi.org/10.1016/j.ecoenv.2017.06.039

Long, J., Cui, Y., Wang, R., Chen, Y., Zhao, N., Wang, C., Wang Z. and Y. Li, 2021. Combined effects of high salinity and ammonia- $\mathrm{N}$ exposure on the energy metabolism, immune response, oxidative resistance and ammonia metabolism of the Pacific white shrimp Litopenaeus vannamei. Aquacul. Rep. 20, 100648. https://doi.org/10.1016/j.aqrep.2021.100648

Lu, X., Kong, J., Luan, S., Dai, P., Meng, X., Cao, B. and Luo K., 2016. Transcriptome analysis of the hepatopancreas in the pacific white shrimp (Litopenaeus vannamei) under acute ammonia stress. PLo S One 11, e0164396. https://doi.org/10.1371/journal.pone.0164396

Ni, Q., Li, W., Jia, X., Dong, Z. and H. Ge, 2020. Effect of salinity on growth performance and resistance of the clam Cyclina sinensis against Vibrio parahaemolyticus infection. Isr. J. Aquacult. Bamid. 72, 1124924. https://doi.org/10.46989/001c.21693

Ni, Q., Li, W., Liang, X., Liu, J., Ge H. and Z. Dong, 2021. Gill transcriptome analysis reveals the molecular response to the acute low-salinity stress in Cyclina sinensis. Aquacul. Rep. 19, 100564. https://doi.org/10.1016/j.aqrep.2020.100564

Oliveira, P., Lírio, A., Canhoto, C. and G. Lúcia, 2018. Toxicity of mercury and postexposure recovery in Corbicula fluminea: Neurotoxicity, oxidative stress and oxygen consumption. Ecol. Indic. 91, 503-510. https://doi.org/10.1016/j.ecolind.2018.04.028 
Rogers, J.A., Metz, L. and V.W. Yong, 2013. Review: endocrine disrupting chemicals and immune responses: a focus on bisphenol-A and its potential mechanisms, Mol. Immunol. 53, 421-430. https://doi.org/10.1016/j.molimm.2012.09.013

Romano N. and C. Zeng, 2007. Acute toxicity of ammonia and its effects on the haemolymph osmolality, ammonia- $\mathrm{N}, \mathrm{pH}$ and ionic composition of early juvenile mud crabs, Scylla serrata (Forskal). Comp. Biochem. Phys. A 148, 278-285.

https://doi.org/10.1016/j.cbpa.2007.04.018

Stebbing A., 1982. Hormesis-the stimulation of growth by low levels of inhibitors, Sci. Total Environ. 22, 213-234. https://doi.org/10.1016/0048-9697(82)90066-3

Wang, X.Q., Wang, L.L., Yao, C., Qiu, L.M., Zhang, H., Zhi, Z. and L.S. Song, 2012. Alternation of immune parameters and cellular energy allocation of Chlamys farreri under ammonia-N exposure and Vibrio anguillarum challenge. Fish shellfish immun. 32(5), 741749. https://doi.org/10.1016/j.fsi.2012.01.025

Xia, Z.Q. and S.J. Wu, 2018. Effects of glutathione on the survival, growth performance and non-specific immunity of white shrimps (Litopenaeus vannamei). Fish Shellfish Immun. 73, 141-144. https://doi.org/10.1016/j.fsi.2017.12.015

Xie, X., Wen, Y., Niu, H., Shi, D. and Z. Zhang, 2012. Re-feeding evokes reproductive overcompensation of food-restricted Brandt's voles. Physiol. Behav. 105 (3), 653-660.

https://doi.org/10.1016/j.physbeh.2011.09.026

Xu, Z., Wang, Y., Gul, Y., Li, Q., Song, J. and M. Hu, 2020. Effects of copper supplement on the immune function and blood-chemistry in adult Chinese horseshoe crab Tachypleus tridentatus.

Aquaculture

515 ,

734576.

https://doi.org/10.1016/j.aquaculture.2019.734576

Yang, W., Xiang, F.H., Liang, L.G. and Z. Yang, 2010. Toxicity of ammonia and its effects on oxidative stress mechanisms of juvenile crucian carp Carassius auratus. J. Freshwater Ecol. 25, 297-302. https://doi.org/10.1080/02705060.2010.9665080

Yang, W., Xiang, F., Sun, H., Chen, Y., Minter, E. and Z. Yang, 2010. Changes in the selected hematological parameters and gill $\mathrm{Na}+/ \mathrm{K}+$ ATPase activity of juvenile crucian carp Carassius auratus during elevated ammonia exposure and the post-exposure recovery. Biochem. Syst. Ecology 38, 557-562. https://doi.org/10.1016/j.bse.2010.06.005

Yuan, Y., Luo, J., Zhu, T., Jin, M., Jiao, L., Sun, P., Ward, T.L., Ji, F., Xu, G. and Q. Zhou, 2020. Alteration of growth performance, meat quality, antioxidant and immune capacity of juvenile Litopenaeus vannamei in response to different dietary dosage forms of zinc: Comparative advantages of zinc amino acid complex. Aquaculture 522, 735120.

https://doi.org/10.1016/j.aquaculture.2020.73512

Yue, F., Pan, L., Xie, P., Zheng, D. and J. Li, 2010. Immune responses and expression of immune-related genes in swimming crab Portunus trituberculatus exposed to elevated ambient ammonia-N stress. Comp. Biochem. Phys. A, 157, 246-251.

https://doi.org/10.1016/j.cbpa.2010.07.013

Zhang, T., Yan, Z., Zheng, X., Fan, J., Wang, S., Wei, Y., Yang, L., Wang, P. and S. Guo, 2019. Transcriptome analysis of response mechanism to ammonia stress in Asian clam (Corbicula fluminea). Aquat. Toxicol. 214, 105235.

https://doi.org/10.1016/j.aquatox.2019.105235 